\title{
El pie equino adquirido en el adulto con encamamiento prolongado
}

\author{
Acquired Equine Foot in Adult in Bed for a Long Time
}

\section{Enrique Tortosa Sirvent ${ }^{1}$}

${ }^{1}$ Grado en Enfermería. enriqueiv@gmail.com

Correspondencia:

Enrique Tortosa Sirvent

Calle Noruega, 7

E-28905 Getafe (Madrid)

Correo electrónico: enriqueiv@gmail.com

Fecha de recepción: 10 de enero de 2011

Fecha de aceptación: 19 de julio de 2011

Los autores declaran no tener ningún tipo de interés económico o comercial.

\section{RESUMEN}

Se hace una revisión bibliográfica con el propósito de conocer el estado actual de conocimientos sobre el pie equino adquirido durante la convalecencia prolongada en cama. Se revisan 32 artículos que arrojan poca evidencia pero de los que se extrae que existen diversos tratamientos, de eficacia limitada, entre los que destaca la cirugía, que parece ser el más efectivo, aunque con reservas. Otros son la fisioterapia, las férulas y elementos ortopédicos. Hay escasos trabajos sobre prevención. Se concluye que es necesario elaborar estudios de mayor calidad y con mayor liderazgo por parte de la enfermería dado el potencial beneficio que pueden recibir los pacientes con esta deformidad.

Palabras clave: pie equino: cuidados críticos; prevención y control; tratamiento.

\section{ABSTRACT}

A bibliographic revision is done in order to know current knowledge about acquired ankle equinus after a long time staying on bed. After revising 32 articles we got little evidence but I found that there are several treatments however, these are not very efficacious. Surgery seems to be the best, but not always. Other treatments are used: physiotherapy, casting and orthopedic elements; although there are very few reports about prevention.

On conclusion, it is needed to make more qualified studies with a major leadership by nursery because its potential benefits to patients who have this deformity.

Key words: Equinus deformity; critic care; prevention and control; treatment.

Referencia normalizada: Tortosa Sirvent, E. El pie equino adquirido en el adulto con encamamiento prolongado. Rev. Int. Cienc. Podol. 2014; 8(1): 61-69.

Sumario: 1. Introducción, 2. Revisión, 3. Resultados, 4. Discusión, 5. Conclusiones, Agradecimientos, Bibliografía. 


\section{INTRODUCCIÓN}

A lo largo del ejercicio de mi profesión como enfermero asistencial y, principalmente, en el ámbito de los cuidados intensivos, yo mismo, como otros compañeros, hemos ido observando la instauración de deformidades en los pies de los pacientes de estancia prolongada que identificamos como pie equino. Problema que, al alta y posterior reincorporación a su vida cotidiana, les ocasiona importantes dificultades en la normal deambulación, impidiéndosela en mayor o menor medida y prolongando los tiempos y costes necesarios de rehabilitación.

Ante esto, muchos de nosotros, sin tener un criterio científico claro, llevábamos a cabo intervenciones como estiramientos pasivos, colocación de almohadas, uso de cajones de madera, etc; con vistas a reducir y/o paliar estas deformidades. La mayor parte de las veces sin efecto y, algunas, incluso, empeorándolas.

El tratar el abordaje del pie equino de manera científica y la búsqueda de unos cuidados basados en la evidencia actual han sido el objeto de llevar a cabo esta revisión.

Para ello, hice una búsqueda bastante exhaustiva en las bases ISI WEB OF KNOWLEDGE, PUBMED, CINAHL, EMBASE, PEDro $\mathrm{y}$ CUIDEN PLUS de los últimos cinco años, criterio que tuve que ampliar a diez y, en algunas ocasiones ir algo más allá al encontrar artículos anteriores de gran utilidad referenciados en otros posteriores. Me serví de las siguientes palabras clave: "ankle equinus", "equinus deformity", "foot drop", "critic care", "prevention and control" y "treatment"; así como sus equivalentes en castellano. Obtuve más de cuarenta artículos de los cuales he terminado seleccionando treinta y dos. Además, tuve el asesoramiento del servicio de rehabilitación del Hospital Universitario de Getafe, lugar donde ejerzo, orientándome en algunos aspectos prácticos.

\section{REVISIÓN}

\subsection{Definición}

Lorimer define pie equino (PE) como "deficiencia funcional en el plano sagital, congénita o adquirida, en la cual existe una limitación en la dorsiflexión de la articulación del tobillo (talocrural)"

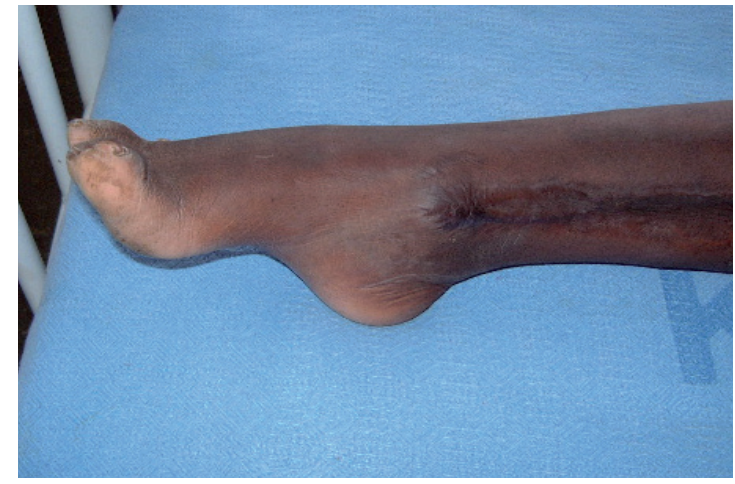

Esta limitación, habitualmente suele ponderarse clínicamente, y de forma más o menos arbitraria, en $10^{\circ}$, que es el ángulo requerido por el individuo para llevar a cabo una deambulación normal, evitando el despegue prematuro del talón cuando se extiende la rodilla al caminar en la fase de apoyo ${ }^{1-6}$. Esta amplitud de $10^{\circ}$ genera controversia ya que, con el pie equino instaurado, la articulación subtalar (la más cercana a la talocrural) puede ejercer un papel compensatorio, bastando entonces un rango de movimiento menor de la articulación talocru$\mathrm{ral}^{4,6,7}$ con lo que podría ser válido un ángulo de $5^{\circ}$ para que el sujeto camine.

\subsection{Determinación diagnóstica}

Este es uno de los aspectos más problemáticos del PE, principalmente en lo que se refiere a la medición del ángulo máximo de dorsiflexión (AMD). Constituye, además, uno de los puntos débiles de los trabajos de investigación, ya que la exactitud en la medición resulta crucial a la hora de comprobar la efectividad de las medidas a estudio. Esto se debe a que no existe un método estandarizado de medida ni una "regla de oro" respecto a la que poder comparar ${ }^{6,7}$. Una técnica precisa resulta costosa y requiere gran cantidad de recursos. Sólo en uno de los estudios revisados se sirven de radiografías seriadas $^{8}$ y lo hacen más bien para validar el resto de mediciones.

No obstante, de la literatura se pueden entresacar ciertos criterios que ha de cumplir una buena medición del AMD:

- Ésta ha de hacerse con el paciente tumbado y la rodilla extendida ${ }^{3}$.

- La articulación del tobillo ha de estar en posición neutra ${ }^{3,6}$. En ese punto mediremos 
el origen del ángulo con los músculos de la pierna relajados ${ }^{8,9}$.

- Aplicaremos una fuerza en la punta de los dedos llevando el pie hacia la tibia todo lo posible (dorsiflexión forzada) $\mathrm{y}$, en ese punto, mediremos el ángulo de la amplitud de movimiento del tobillo (ángulo comprendido entre la línea media del peroné y el quinto metatarsiano) $)^{6,8}$. Esta fuerza debería estar estandarizada pero en los estudios no suele ser así. Digiovanni la fija en $10 \mathrm{~N}-\mathrm{m}^{7}$.

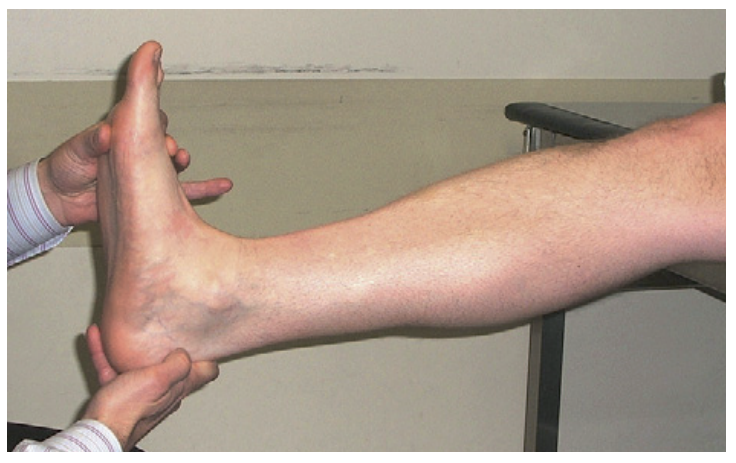

El método de medición más extendido es mediante goniómetro que es, además, el más sencillo y barato. No obstante, su fiabilidad es discutida $^{10}$. Por contra, el más preciso parece ser el equinómetro ${ }^{7,11}$, artefacto creado expresamente para esta función pero que requiere formación para su correcto empleo, gran cantidad de recursos materiales, resulta caro y complicado de usar.

\subsection{Clasificación}

Varios autores proponen una clasificación funcional del pie equino basada en aquellos parámetros de movilidad expuestos en el punto 1.1., a saber ${ }^{1,6}$ :

- Estadio I: Dorsiflexión menor de $10^{\circ}$ $\left(\mathbf{1 0}^{\circ}>\mathbf{x}>5^{\circ}\right)$, indicando menores cambios adaptativos y menor incremento de la presión en la zona delantera del pie.

- Estadio II: Dorsiflexión menor o igual a $5^{\circ}\left(5^{\circ} \geq \mathbf{x}\right)$, indicando mayores cambios adaptativos y mayor incremento de la presión en la zona delantera del pie.

Sin embargo, la clasificación que de forma más común solemos encontrar se refiere a su etiología: primario (congénito) o secundario (adquirido) y sus correspondientes subtipos $^{5}$ (Figura 1).

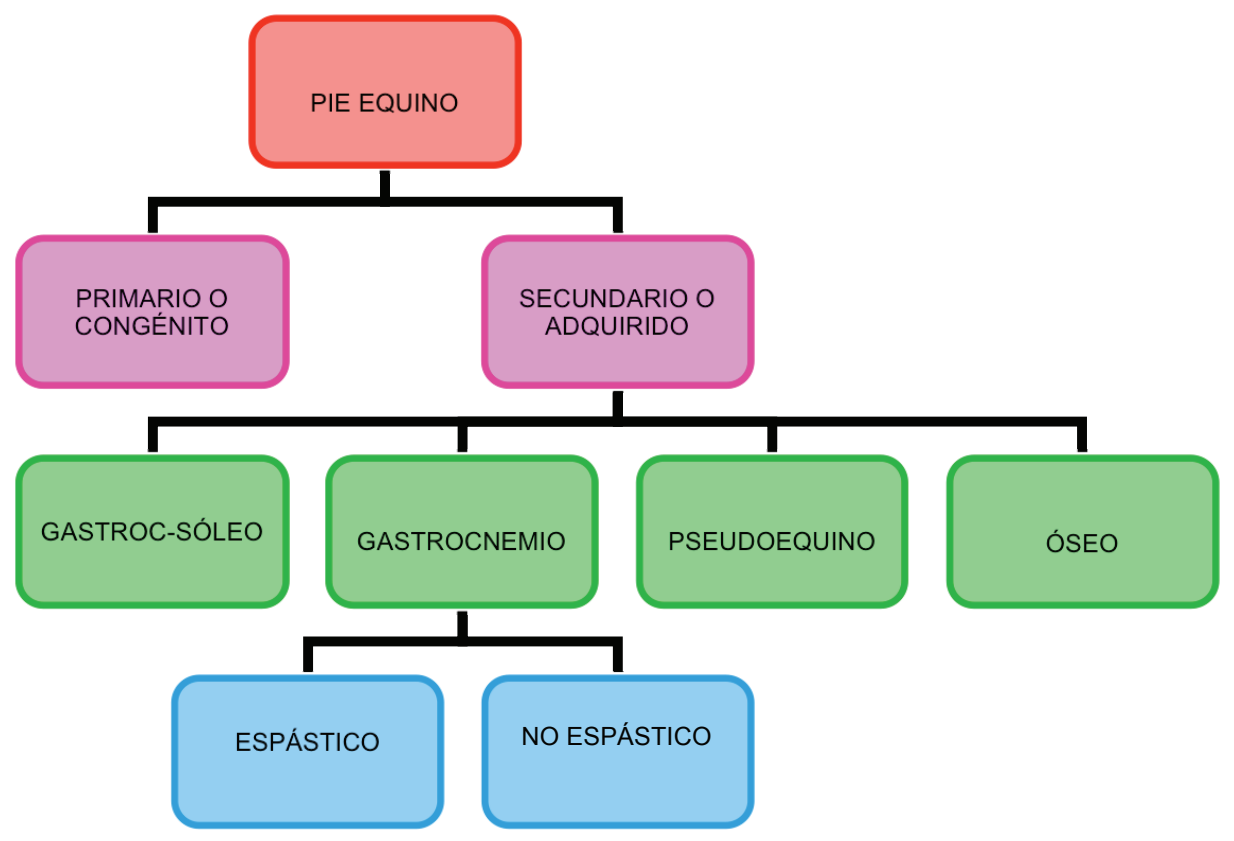

Figura 1. Clasificación del pie equino por su etiología. 
Teniendo en cuenta esta última categorización, me centraré en el pie equino adquirido, campo en el que el trabajo enfermero tiene mucho que decir y donde aplicar unos cuidados de calidad puede redundar enormemente en el beneficio de los pacientes a nuestro cargo.

\subsection{Etiología y factores relacionados}

En el ámbito hospitalario, y más concretamente en el de los cuidados intensivos, es frecuente tratar con pacientes que sufren un encamamiento prolongado, afectación del nivel de conciencia, tratamientos con fármacos sedantes y relajantes y, muchas veces, además, con problemas neuromusculares ${ }^{12,13}$. Estos factores terminan derivando en un síndrome de desuso, y la consecuente debilidad de los músculos dorsiflexores del tobillo ${ }^{15}$. Ocasionándolo y agravándolo a la vez.

No obstante, el origen del PE no está nada claro $^{6}$. Para su explicación se ha recurrido a estudios en animales. Varios de ellos han descrito cambios morfológicos tras inmovilizaciones prolongadas tales como acortamiento de músculos y tendones por una disminución del número de sarcómeros de las fibras que los componen, además de la elongación de los músculos antagonistas por adición de nuevo material (sarcogénesis). También hablan de un descenso en la elasticidad de los tejidos blandos periarticulares hasta, con el paso del tiempo, hacer estas alteraciones definitivas por crecimiento de fibras de colágeno (leyes de Davis, Delpech y Wolf). Todo ello conlleva el acortamiento progresivo del tendón de Aquiles (por parte del gastrocnemio, principalmente, y por el gastrocsóleo) así como atrofia del tríceps sural $1^{1-3,6,8,9,12,13,16-19}$, que da al PE su característica forma limitando su movilidad. Con frecuencia se rota hacia el interior y aparece el equinovaro ${ }^{19}$.

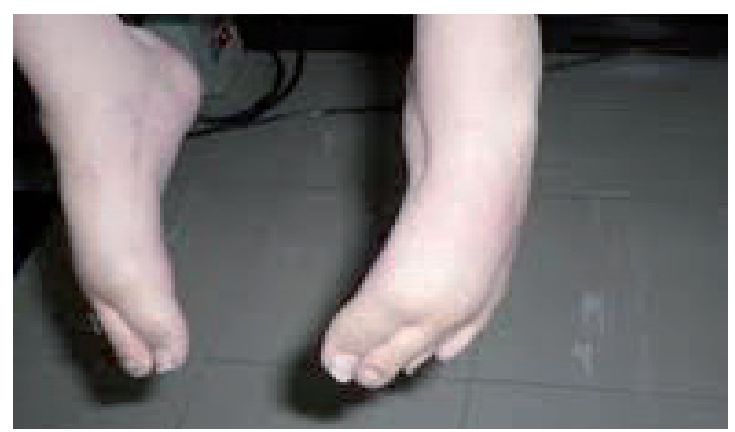

Conjuntamente, se pueden también asociar fenómenos de contractura, compresión nerviosa por malposición, espasticidad, hipertonía e hiperreflexia, muy prevalentes en pacientes con patologías neurológicas y neuromusculares, entroncándose frecuentemente, e incluso confundiéndose con otra entidad de aspecto externo semejante pero de origen y tratamiento distinto: el pie caído o foot drop. . $^{210,14,15,19,20}$

Expósito fija unos 8 días de plazo para la instauración del $\mathrm{PE}^{8}$ pero no da referencias en las que basarse. Lehmkuhl unas pocas semanas $^{21}$. Ningún otro de los artículos revisados dice nada acerca de ello.

En consideración de lo anterior, se han descrito diversos factores relacionados con el PE:

- Edad. Se ha visto una reducción de la dorsiflexión en pacientes ancianos sin patología previa ${ }^{16}$.

- Pacientes con Accidente Cerebro-Vascular $(\mathrm{ACV})$ y patología neurológica en general $2^{2,6,11,12,15,19,22}$. Es, de hecho, la deformidad más común asociada a hipertonicidad en este tipo de pacientes ${ }^{23}$.

- Traumatismos y lesiones periarticulares $^{1,21,22}$.

- Enfermedades neuromusculares a nivel de la segunda motoneurona ${ }^{15,17}$.

- Niveles altos de glucosa en pacientes diabéticos ${ }^{6}$, por alteraciones en las proteínas del tejido conectivo.

En cuanto al uso de fármacos sedantes y relajantes, no he encontrado a nadie que haya estudiado directamente su influencia. Expósito parece afirmar que no influye en el $\mathrm{AMD}^{8}$. Si bien, parece probable que influyan indirectamente, por las consecuencias de su uso.

\subsection{Magnitud y costes}

A pesar de la frecuencia con la que viene descrito en los distintos estudios, apenas hay datos globales sobre la incidencia o prevalencia del PE. Apenas alguna reseña y siempre en patologías muy concretas. Tras consultar al Servicio de Fisioterapia y Rehabilitación de Hospital Universitario de Getafe, me aseguraron no contar con ningún registro específico de los $\mathrm{PE}$ tratados al cabo del año. Lo que sí parece estar claro es que, a mayor duración del coma, ma- 
yor el número de articulaciones contracturadas y mayor el número de $\mathrm{PE}^{19}$.

Singer da la cifra de un $76 \%$ tras un año pero refiriéndose a enfermos de trauma cerebral ${ }^{2,9}$. En una revisión, Sackley, citando a Geboers, establece un caso por cada 6000 al año atendido en un servicio de rehabilitación de Holanda aunque él mismo reconoce que puede ser una cifra errónea ${ }^{14}$. Meszaros maneja hasta la presencia del PE en un 96\% de los pacientes con dolor inducido por causas biomecánicas en la pisada ${ }^{5}$.

En el otro extremo, Verdié plantea un 18\% en pacientes supervivientes de ACV y tras un año de evolución ${ }^{24}$.

La gran variabilidad de cifras encontradas puede deberse a la dificultad en establecer un diagnóstico adecuado, en utilizar muestras pequeñas $y$, probablemente, por considerar el PE dentro de entidades mayores y no contabilizando el número de $\mathrm{PE}$ totales estén ligados a la patología que sea.

Lo que sí parece claro son las muchas consecuencias para el enfermo de padecer un PE: dificultades en la marcha, limitación para ponerse de pie, subir escaleras, cambiarse de asiento, inestabilidad y mayor riesgo de esguinces ${ }^{6}$, caídas $^{9,13,16,17,21}$, dolor, sobrecarga en la parte anterior del pie, metatarsalgia y fascitis plan$\operatorname{tar}^{17}$, dolor de rodillas, tensión en la zona lumbar y lordosis, fatiga muscular y hasta genu recurvatum por las compensaciones adaptativas necesarias ${ }^{5}$. Aspectos que dificultan, e incluso limitan la actividad y vida productiva diaria de los sujetos. También tiene una prevalencia significativa en individuos con úlceras plantares ${ }^{11}$.

En el aspecto económico, Abrisqueta asevera que se da un aumento del tiempo de estancia de los enfermos y del gasto por un retraso en la recuperación aunque, si bien no da ningún dato que lo apoye, parece bastante plausible ${ }^{13}$. Lehmkuhl cifra en unos 900 \$ el gasto en férulas entre material y profesionales para el tratamiento $^{21}$.

\subsection{Tratamiento}

En la actualidad existe un buen número de tratamientos para el PE. No obstante, la mayoría de los autores consultados se ponen bastante de acuerdo en algo: hay muy poca evidencia que apoye la superioridad de uno sobre otro y, en general, la efectividad no es alta. La elección de los tratamientos suele hacerse más por opinión de expertos y experiencias personales que apoyándose en unos estudios que, en su mayoría, son de poca calidad ${ }^{3,17,18,25}$. Sí que parece que existe una tendencia a combinarlos y abordarlos desde una perspectiva multidisciplinar de enfermeros, fisioterapeutas, podólogos, ortopedistas, traumatólogos, rehabilitadores y terapeutas ocupacionales visto el amplio abanico de profesionales que aportan sus posibles soluciones.

Lo primero que se recomienda es determinar de forma certera el origen del PE. El test de Silfverskiold (si AMD $<10^{\circ}$ con rodilla estirada y flexionada, origen gastrocnemio y gastrocsóleo; si $\mathrm{AMD}<10^{\circ}$ sólo con rodilla estirada, origen gastrocnemio), nos ayuda a discriminar cuáles pudieran ser los músculos implicados $\mathrm{y}$, una vez establecido se aplican los siguientes tratamientos ${ }^{1-3,15}$ :

\subsubsection{Férulas y fármacos asociados}

Es uno de los más utilizados. Se usan férulas estándar y fabricadas a medida de diversos materiales como yeso y fibra de vidrio. Pueden ser bivalvas o sencillas y se aplican de múltiples maneras diferentes. Hay férulas nocturnas y tratamientos seriados con férulas que fuerzan cada vez a una mayor dorsiflexión ${ }^{9,14,19,21}$ aunque hay poco consenso en el régimen horario a usar y el tiempo necesario para producir sus efectos.

$\mathrm{Su}$ empleo se basa en el favorecimiento de la sarcogénesis antes comentada. Además, parece reducir la hipertonía e hiperreflexia ${ }^{10,23,26}$. Tal vez el calor proporcionado por el material pueda servir como coadyuvante ${ }^{17}$.

En algunos casos se plantea el dilema de si la propia férula empeora el desuso de los músculos y la debilidad aunque el trabajo de Geboers lo desestima ${ }^{27}$, si bien, se basa en pacientes con pie caído. La férula ideal debería permitir un normal movimiento, limitando la plantaflexión y favoreciendo la dorsiflexión, dejando, además, un buen acceso a la pierna ${ }^{28}$.

Entre los problemas descritos con el uso de férulas están: irritación y ulceración de la piel, tendinitis, rigidez, edema, compresión de tejidos circundantes, dolor ${ }^{17,18,21,23}$. 
Se asocia a veces con toxina botulínica empleada de forma $\operatorname{local}^{29} \mathrm{y}$, en pacientes con ciertas enfermedades neuromusculares, con corticoides. En los dos casos con poca evidencia de mejoría y sí con aumento del gasto y los efectos indeseables ${ }^{2,17,18}$.

\subsubsection{Fisioterapia}

Englobo aquí tratamientos basados en masajes y estiramientos así como los que se basan en elementos electrofisiológicos como el calor (efecto dilatador de la fibra) y la estimulación eléctrica ${ }^{17}$.

La mayoría trata de aumentar el AMD, reducir la espasticidad y la contractura mediante estiramientos y maniobras ${ }^{1,3,8,17,19,23,30}$ así como programas de entrenamiento para incrementar la fuerza de los dorsiflexores ${ }^{14-16}$. Ningún artículo revisado detalla en qué consisten las maniobras ni la frecuencia con la que las realiza, excepto Dananberg, que relaciona el PE con una falta de traslación de la cabeza del peroné, hecho que consigue paliar mediante las manipulaciones que describe. Sólo él ha publicado esta relación y el artículo no parece muy válido pero puede aportar nuevas líneas de investigación.

Por otro lado, cada vez parece más habitual el empleo de la estimulación eléctrica, no sólo para aumentar la fuerza de los músculos sino también para reducir la espasticidad ${ }^{31}$.

Muchas de estas terapias se usan con frecuencia en asociación con férulas y tras procedimientos invasivos que veremos más adelante. Los trabajos incluidos sobre métodos fisioterapéuticos no dan una gran evidencia de la utilidad como elemento curativo de manera aislada, sin embargo, sí que son los que más en cuenta tienen el estudio sobre ganancia de independencia para las actividades de la vida diaria ${ }^{14,16}$.

Como desventajas de su empleo está el dolor y la posibilidad de exacerbar el equino por estimulación de los reflejos tendinosos ${ }^{23}$.

\subsubsection{Elementos ortopédicos y fijadores ex- ternos (Dispositivo de Ilizarov)}

Se trata de un grupo bastante heterogéneo que recoge ortosis, zapatos ortopédicos y con modificaciones e, incluso, fijadores externos percutáneos tipo dispositivo de Ilizarov. Su acción se fundamenta básicamente en la corrección progresiva de la deformidad $d^{1,3,8,15,22}$. Han de uti- lizarse de manera muy prolongada y, en el caso de los zapatos ortopédicos y modificados a veces se prefieren por ser más discretos ${ }^{25}$. No es el caso del dispositivo de Ilizarov, que resulta sumamente aparatoso. Sin embargo, el modificado por Melvin y Dahners parece ofrecer buenos resultados a un año vista ${ }^{15,22}$.

Como efectos adversos podemos encontrar deformidad de los tejidos blandos, si no se colocan correctamente y dolor ${ }^{22}$.

\subsubsection{Tratamiento quirúrgico}

De todos los tratamientos, parece ser el más efectivo y el que tiene mayores ventajas según van Til ${ }^{25}$. Habitualmente se opta por él cuando el tratamiento conservador fracasa ${ }^{2}$. En la revisión de Rose, a pesar de que es el procedimiento que más incrementa el AMD, éste va disminuyendo según pasan los meses y no parece haber mejoría en fuerza muscular y en función ${ }^{14,17}$. Sin embargo, sus estudios son sobre patología neuromuscular y mayormente en niños.

Las técnicas utilizadas son varias. Abarcan las transposiciones tendinosas y el alargamiento y acortamiento de músculos según sea el origen de la deformidad junto con las propias preferencias del cirujano, ya que no hay mucha evidencia de superioridad de una sobre las demás ${ }^{5,22}$.

Como desventajas encontramos las complicaciones propias de la cirugía, dehiscencia de heridas, infección, rotura de tendones dolor, molestias relacionadas con presión del calzado sobre las cicatrices ${ }^{17,32}$.

Una buena rehabilitación postquirúrgica es imprescindible para alcanzar los objetivos previstos $^{15}$.

\subsection{Prevención}

Todo lo visto hasta ahora pone de manifiesto la trascendencia que tiene el PE en los pacientes que atendemos y cómo éste va a condicionar su recuperación.

Es importantísima la prevención ${ }^{14}$ pero, curiosamente, se dedica gran cantidad de dinero, tiempo, personal y producción científica a la búsqueda y aplicación de tratamientos. En lo que respecta a la prevención, apenas se encuentra nada. Poquísimos artículos que, además, aportan escasa evidencia ${ }^{2,8,17}$ se ocupan de esto.

Este es el campo más propio de la enfermería, donde puede y debe desempeñar un papel 
crucial como proveedor de cuidados continuos y evitando o reduciendo, en la medida de lo posible, la instauración de un PE. De hecho, casi todos los artículos sobre prevención son publicados por enfermeros

La medida más habitual de prevención es el empleo de férulas antiequino. Lo ideal es que estén fabricadas a medida, que no lleguen al hueco poplíteo y que mantengan el tobillo en posición neutra o ligera dorsiflexión ${ }^{21}$. Estas férulas han de estar colocadas mientras el enfermo esté en cama, especialmente si está sedado o en coma, siguiendo una rutina diaria con períodos de descanso para evitar la rigidez, la incomodidad y la aparición de úlceras. Resulta útil la colocación de almohadas en los laterales de las piernas para impedir la rotación de las caderas y miembros inferiores ${ }^{12}$. En los períodos de descanso, se recomienda la hidratación de la piel, la movilización activa (si el enfermo puede colaborar) y pasiva, estiramientos y la liberación de la presión sobre los talones y el tendón de Aquiles ${ }^{8,13-15}$. Las pautas propuestas por los trabajos consultados no aportan evidencia ni pruebas de mayor idoneidad de unas sobre otras. El artículo de Expósito, aun con sus defectos, presenta unos resultados alentadores en cuanto a efectividad ${ }^{8}$. Plantea un régimen de períodos de seis horas con las férulas colocadas y dos horas de descanso en las que se llevan a cabo las medidas arriba comentadas.

Según el trabajo de Hyde, revisado por Rose, se produce un retraso en la aparición de la contractura del tobillo al emplear férulas nocturnas en niños aquejados de enfermedad neuromuscular $^{17}$.

Otros medios a emplear pueden ser arneses y vendajes antiequinos, que colocan el pie en ángulo recto ${ }^{13}$. Incluso, existe un protocolo presentado en congreso del 2009 de la SEEIUC de la UCI del hospital Germans Trias i Pujol de Badalona en el que utilizan zapatillas de lona “tipo Converse ${ }^{\circledR}$ ” dos números mayores que el pie del paciente y rellenas con algodón pero no me han facilitado datos acerca de sus resul$\operatorname{tados}^{12}$.

La explicación de estos métodos podría residir en la reducción de la hipertonía y la caída de la parte anterior del pie debido a la gravedad, así como la debilidad muscular y la contractura del tendón de Aquiles ${ }^{14,15,21}$.
Queda excluido su uso en pacientes con patología vascular, edemas distales, úlceras o malformaciones ${ }^{12}$.

\section{DISCUSIÓN}

Este es un aspecto del trabajo que prácticamente ya he venido realizando en los epígrafes anteriores, así que sólo haré unas someras apreciaciones.

El principal problema de los estudios revisados es su validez y baja calidad general. En algunos casos no se define bien el criterio diferenciador entre equino y no equino. En su mayoría trabajan con muestras bastante pequeñas y pocas veces hacen análisis de homogeneidad al compararlas. No he encontrado estudios realmente aleatorios. Además, hay un importantísimo problema con la medición del AMD, que puede condicionar absolutamente todo. Casi todos los autores usan un goniómetro (con las limitaciones ya comentadas) o no describen la técnica. Sólo en un caso se usan radiografías de control.

Rara vez hay "ciegos" entre los participantes del estudio. Es cierto que en ocasiones se usan dispositivos difíciles de ocultar pero en muchos artículos se podía haber intentado algún cegamiento.

Parte de las revisiones más rigurosas consultadas, también incluyen estudios con niños, con lo que hay que considerar de manera aproximada las conclusiones, puesto que parece que la edad influye en el establecimiento de un PE.

A veces, tampoco se aportan razones de por qué se aplica una pauta terapéutica en lugar de otra diferente.

Finalmente, destacar que hay pocos trabajos que sigan a sus sujetos de estudio a lo largo del tiempo, la consecución de tareas propias de la vida diaria y el autocuidado. En los que sí que lo han hecho se ha visto un progresivo retroceso en el AMD con lo que la efectividad de un tratamiento se pone en entredicho.

\section{CONCLUSIONES}

Tras la revisión efectuada, se echan en falta trabajos de mayor calidad que aporten más 
evidencia, puesto que ahora mismo se cuenta con muy poca, sobre un problema que se ve con bastante frecuencia y al que se le dedican muchos recursos. Los tratamientos actuales son poco efectivos y se dedica escasa atención a la prevención. Pienso que hay muchas posibles líneas de investigación en este ámbito en el que la enfermería es imprescindible, dada su cercanía con el enfermo y su labor de agente de cuidados básicos para con un paciente que no puede proporcionárselos a sí mismo.

\section{AGRADECIMIENTOS}

Quería finalmente expresar mi agradecimiento a Ana Gómez Morejudo, fisioterapeuta del Hospital Universitario de Getafe por su ayuda y desinterés en la elaboración de este trabajo.

\section{BIBLIOGRAFÍA}

1. Lorimer D, Neale D, French G. Neale's disorders of the foot: diagnosis and management. $6^{\mathrm{a}}$ ed.: Churchil Livingstone; 2001. p. 123-123-125;348-349.

2. 2.Singer BJ, Dunne JW, Singer KP, Jegasothy GM, Allison GT. Non-surgical management of ankle contracture following acquired brain injury. Disabil Rehabil 2004 Mar 18;26(6):335-345. http://dx.doi. org $/ 10.1080 / 0963828032000174070$

3. 3.Chen L, Greisberg J. Achilles lengthening procedures. Foot Ankle Clin 2009 Dec;14(4):627-637. http://dx.doi.org/10.1016/j.fcl.2009.08.002

4. 4.Weir J, Chockalingam N. Ankle joint dorsiflexion: assessment of true values necessary for normal gait. Int J Ther Rehabil 2007 02;14(2):76-82.

5. 5.Meszaros A, Caudell G. The surgical management of equinus in the adult acquired flatfoot. Clin Podiatr Med Surg 2007 Oct;24(4):667-85, viii. http://dx.doi.org/10.1016/j.cpm.2007.07.007

6. 6.Charles J, Scutter SD, Buckley J. Static ankle joint equinus: toward a standard definition and diagnosis. J Am Podiatr Med Assoc 2010 May-Jun;100(3):195-203.

7. 7.Digiovanni CW, Holt S, Czerniecki JM, Ledoux WR, Sangeorzan BJ. Can the presence of equinus contracture be established by physical exam alone? J Rehabil Res Dev 2001 May-Jun;38(3):335-340.

8. 8.Exposito A. El pie equino adquirido en el paciente de UCI. Revista Española de Podología 1997; VIII(2):84-101.

9. 9.Moseley AM. The effect of casting combined with stretching on passive ankle dorsiflexion in adults with traumatic head injuries. Phys Ther 1997 Mar;77(3):240-7; discussion 248-59.

10. 10.Mortenson PA, Eng JJ. The use of casts in the management of joint mobility and hypertonia following brain injury in adults: a systematic review. Phys Ther 2003 Jul;83(7):648-658.

11. 11.Assal M, Shofer JB, Rohr E, Price R, Czerniecki J, Sangeorzan BJ. Assessment of an electronic goniometer designed to measure equinus contracture. J Rehabil Res Dev 2003 May-Jun;40(3):235-239.

12. 12.Casanovas C. Prevenció del peu equí. Utilització de calçat. Badalona: Hospital Germans Trias i Pujol; 2010. INF-PI-197.

13. 13.Abrisqueta L, Carmona Simarro JV, Montáñez FJ. Prevención del pie equino en la unidad de críticos. Enfermería Integral 2005(70):9-12.

14. 14.Sackley C, Disler PB, Turner-Stokes L, Wade DT, Brittle N, Hoppitt T. Rehabilitation interventions for foot drop in neuromuscular disease (Cochrane review) [with consumer summary]. Cochrane Database of Systematic Reviews 2009(3). http://dx.doi.org/10.1002/14651858.CD003908.pub3

15. 15.Jaivin JS, Bishop JO, Braly WG, Tullos HS. Management of Acquired Adult Dropfoot. Foot Ankle 1992 FEB;13(2):98-104.

16. 16.Kluding PM, Santos M. Effects of ankle joint mobilizations in adults poststroke: a pilot study. Arch Phys Med Rehabil 2008 Mar;89(3):449-456. http://dx.doi.org/10.1016/j.apmr.2007.12.005

17. 17.Rose KJ, Burns J, Wheeler DM, North KN. Interventions for increasing ankle range of motion in patients with neuromuscular disease (Cochrane review) [with consumer summary]. Cochrane Database of Systematic Reviews 2010(2). http://dx.doi.org/10.1002/14651858.CD006973.pub2

18. 18.Blackmore AM, Boettcher-Hunt E, Jordan M, Chan MD. A systematic review of the effects of casting on equinus in children with cerebral palsy: an evidence report of the AACPDM. Dev Med Child Neurol 2007 Oct;49(10):781-790. http://dx.doi.org/10.1111/j.1469-8749.2007.00781.x 
19. 19.Watkins CA. Mechanical and neurophysiological changes in spastic muscles. Physioterapy 1999 november;85(11):599-605.

20. 20.Latronico N, Shehu I, Seghelini E. Neuromuscular sequelae of critical illness. Curr Opin Crit Care 2005 Aug;11(4):381-390.

21. 21.Lehmkuhl LD, Thoi LL, Baize C, Kelley CJ, Krawczyk L, Bontke CF. Multimodality treatment of joint contractures in patients with severe brain injury: Cost, effectiveness, and integration of therapies in the application of serial/inhibitive casts. J Head Trauma Rehabil 1990;5(4):23-42.

22. 22.Mendicino RW, Murphy LJ, Maskill MP, Catanzariti AR, Penny H. Application of a constrained external fixator frame for treatment of a fixed equinus contracture. J Foot Ankle Surg 2008 SepOct;47(5):468-475. http://dx.doi.org/10.1053/j.jfas.2008.05.011

23. 23.Conine TA, Sullivan T, Mackie T, Goodman M. Effect of serial casting for the prevention of equinus in patients with acute head injury. Arch Phys Med Rehabil 1990 Apr;71(5):310-312.

24. 24.Verdié C, Daviet JC, Borie MJ, Popielarz S, Munoz M, Salle JY, et al. Épidémiologie des pieds varus et/ou équin un an après un premier accident vasculaire cérébral hémisphérique: à propos d'une cohorte de 86 patients. Ann Readapt Med Phys 2004 Mar;47(2):81-86. http://dx.doi.org/10.1016/j.annrmp.2003.10.005

25. 25.van Til JA, Renzenbrink GJ, Dolan JG, Ijzerman MJ. The use of the analytic hierarchy process to aid decision making in acquired equinovarus deformity. Arch Phys Med Rehabil 2008 Mar;89(3):457462. http://dx.doi.org/10.1016/j.apmr.2007.09.030

26. 26.Geboers JF, Drost MR, Spaans F, Kuipers H, Seelen HA. Immediate and long-term effects of anklefoot orthosis on muscle activity during walking: a randomized study of patients with unilateral foot drop. Arch Phys Med Rehabil 2002 Feb;83(2):240-245. http://dx.doi.org/10.1053/apmr.2002.27462

27. 27.Geboers JF, Janssen-Potten YJ, Seelen HA, Spaans F, Drost MR. Evaluation of effect of ankle-foot orthosis use on strength restoration of paretic dorsiflexors. Arch Phys Med Rehabil 2001 Jun;82(6):856860. http://dx.doi.org/10.1053/apmr.2001.21863

28. 28.Chin R, Hsiao-Wecksler E, Loth E, Kogler G, Manwaring S, Tyson S, et al. A pneumatic power harvesting ankle-foot orthosis to prevent foot-drop. Journal of NeuroEngineering and Rehabilitation 2009;6(1):19. http://dx.doi.org/10.1186/1743-0003-6-19

29. 29.Bayram S, Sivrioglu K, Karli N, Ozcan O. Low-dose botulinum toxin with short-term electrical stimulation in poststroke spastic drop foot: a preliminary study. Am J Phys Med Rehabil 2006;85(1):7581.

30. 30.Dananberg HJ, Shearstone J, Guillano M. Manipulation method for the treatment of ankle equinus. J Am Podiatr Med Assoc 2000 Sep;90(8):385-389.

31. 31.Ijzerman MJ, Renzenbrink GJ, Geurts ACH. Neuromuscular stimulation after stroke: from technology to clinical deployment. Expert Rev Neurotherapeutics 2009 04/01; 2011/01;9(4):541-552. http:// dx.doi.org/10.1586/ern.09.6

32. 32.Sammarco GJ, Bagwe MR, Sammarco VJ, Magur EG. The effects of unilateral gastrocsoleus recession. Foot Ankle Int 2006 07;27(7):508-511. 